\title{
Cutaneous Manifestations and Clinical Disparities in Patients Without Housing
}

\author{
Marguerite O'Quinn, BS; Christopher Haas, MD; Deborah Hilton, MD
}

\section{PRACTICE POINTS}

- Dermatologic disease in patients without housing $(\mathrm{NWH})$ is characterized by more infectious concerns and fewer follicular and urticarial noninfectious inflammatory eruptions compared with matched controls of those with housing.

- Patients with housing more frequently presented with cutaneous chief concerns and received more consu tations while in the hospital.

- This study uncovered notable pathological and clinical differences in treating dermatologic conditions in $\mathrm{NWH}$ patients.

Dermatologic disease in patients without housing (NWH) has not been well characterized. We present a retrospective cohort study delineating dermatologio disease in $\mathrm{WWH}$ patients and patients with housing $(\mathrm{WH})$ during presentation to the emergency department. A total of 842 medical records were reviewed, with evenly matched $\mathrm{NWH}$ and WH patients based on sex, self-identified race and ethnicity, and age. To improve outcomes in this vulnerable population, our study sought to elucidate more information on the morphology of cutaneous disease and highlight disparities in clinical workup.

Cutis. 2021;108:222-226.

M ore than half a million individuals are without housing (NWH) on any given night in the United States, as estimated by the US Department of
Housing and Urban Development. ${ }^{1}$ Lack of hygiene, increased-risk infection and infestation due to living conditions, and barriers to health care put these individuals at increased risk for disease. ${ }^{2}$ Skin disease, including fungal infection and acne, are within the top 10 most prevalent diseases worldwide and can cause major psychologic impairment, yet dermatologic concerns and clinical outcomes in NWH patients have not been well characterized. ${ }^{2-5}$ Further, because this vulnerable demographic tends to be underinsured, they frequently present to the emergency department (ED) for management of disease. ${ }^{1,6}$ Survey of common concerns in NWH patients is of utility to consulting dermatologists and nondermatologist providers in the ED, who can familiarize themselves with management of diseases they are more likely to encounter. Few studies examine dermatologic conditions in the $\mathrm{ED}$, and a thorough literature review indicates none have included homelessness as a variable. ${ }^{6,7}$ Additionally, comparison with a matched control group of patients with housing $(\mathrm{WH})$ is limited. ${ }^{5,8} \mathrm{We}$ present one of the largest comparisons of cutaneous disease in NWH vs WH patients in a single hospital system to elucidate the types of cutaneous disease that motivate patients to seek care, the location of skin disease, and differences in clinical care.

\section{Methods}

A retrospective medical record review of patients seen for an inclusive list of dermatologic diagnoses in the ED or while admitted at University Medical Center

From Louisiana State University Health Sciences Center New Orleans. Ms. O'Quinn is from the School of Medicine. Drs. Haas and Hilton are from the Department of Dermatology.

The authors report no conflicts of interest.

Correspondence: Marguerite O'Quinn, BS, 1524 Tulane Ave, Ste 639, New Orleans, LA 70112 (moqui1@lsuhsc.edu).

doi:10.12788/cutis.0367 
New Orleans, Louisiana (UMC), between January 1, 2018, and April 21, 2020, was conducted. This study was qualified as exempt from the institutional review board by Louisiana State University because it proposed zero risk to the patients and remained completely anonymous. Eight hundred forty-two total medical records were reviewed (NWH, 421; WH, 421)(Table 1). Patients with housing were matched based on self-identified race and ethnicity, sex, and age. Disease categories were constructed based on fundamental pathophysiology adapted from Dermatology': infectious, noninfectious inflammatory, neoplasm, trauma and wounds, drug-related eruptions, vascular, pruritic, pigmented, bullous, neuropsychiatric, and other. Other included unspecified eruptions as well as miscellaneous lesions such as calluses. The current chief concern, anatomic location, and configuration were recorded, as well as biopsied lesions and outpatient referrals or inpatient consultations to dermatology or other specialties, including wound care, infectious disease, podiatry, and surgery. $\chi^{2}$ analysis was used to analyze significance of cutaneous categories, body location, and referrals. Groups smaller than 5 defaulted to the Fisher exact test.

\section{Results}

The total diagnoses (including both chief concerns and secondary diagnoses) are shown in Table 2. Chief concerns were more frequently cutaneous or dermatologic for WH (NWH, 209; WH, 307; P<.001). In both groups, cutaneous infectious etiologies were more likely to be a patient's presenting chief concern $(58 \% \mathrm{NWH}, P=002$; $42 \% \mathrm{WH}, P<.001)$. Noninfectious inflammatory etiologies and pigmented lesions were more likely to be secondary diagnoses with an undelated noncutaneous concern; noninfectious inflammatory etiologies were only $16 \%$ of the total cutaneous chief concerns $(11 \% \mathrm{NWH}$,

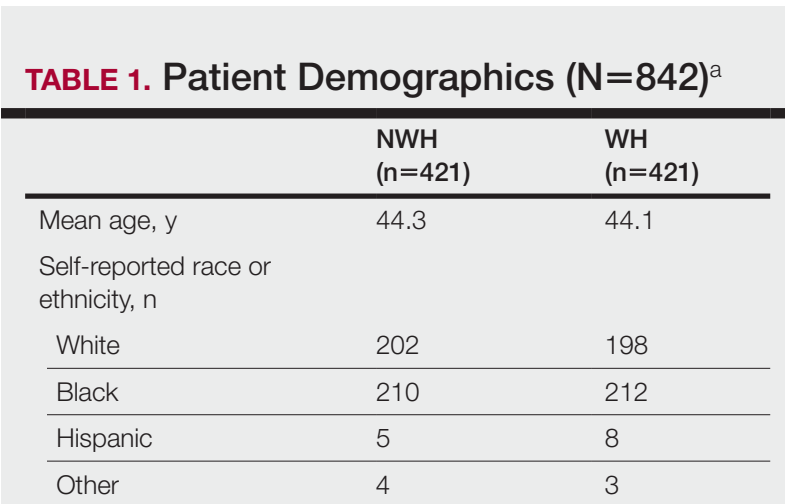

Sex, $\mathrm{n}$

\begin{tabular}{lll} 
Male & 308 & 306 \\
\hline Female & 113 & 115 \\
\hline
\end{tabular}

Abbreviations: $\mathrm{NWH}$, without housing; $\mathrm{WH}$, with housing

There were no significant differences between $\mathrm{NWH}$ and WH patients.
$P=.04 ; 20 \% \mathrm{WH}, P=.03)$, and no pigmented lesions were chief concerns.

Infection was the most common chief concern, though $\mathrm{NWH}$ patients presented with significantly more infectious concerns (NWH, 212; WH, 150; $P<.001)$, particularly infestations $(\mathrm{NWH}, 33 ; \mathrm{WH}, 8 ; P<.001)$ and bacterial etiologies (NWH, 127; WH, 100; $P=.04$ ). The majority of bacterial etiologies were either an abscess or cellulitis ( $\mathrm{NWH}, 106 ; \mathrm{WH}, 83)$, though infected chronic wounds were categorized as bacterial infection when treated definitively as such (eg, in the case of sacral ulcers causing osteomyelitis) $(\mathrm{NWH}, 21 ; \mathrm{WH}, 17)$. Of note, infectious etiology was associated with intravenous drug use (IVDU) in both NWH and WH patients. Of $184 \mathrm{NWH}$ who reported IVDU, 127 had an infectious diagnosis $(P<.001)$. Similarly, 43 of 56 total WH patients who reported IVDU had an infectious diagnosis $(P<.001)$. Infestation (within the infectious category) included scabies (NWH, 20, WH, 3) and insect or arthropod bites ( $\mathrm{NWH}, 12 ; \mathrm{WH}, 5)$. Two $\mathrm{NWH}$ patients also presented with swelling of the lower extremities and were subsequently diagnosed with maggot infestations. Fungal and viral etiologies were not significantly increased in either group; however, NWH did have higher incidence of tinea pedis $(\mathrm{NWH}, 14$; W $\mathrm{H}, 4 ; \mathrm{P}=.03$ ).

More neoplasms (NWH, 6; WH, 16; $P=.03$ ), noninfectious inflammatory eruptions $(\mathrm{NWH}, 48 ; \mathrm{WH}$, 85; $P<.001)$, and cutaneous drug eruptions $(\mathrm{NWH}, 5$; $\mathrm{WH}, 27 ; P<.001)$ were reported in $\mathrm{WH}$ patients. There was no significant difference in benign vs malignant neoplastic processes between groups. More noninfectious inflammatory eruptions in $\mathrm{WH}$ were specifically driven by a markedly increased incidence of follicular (NWH, 9; $\mathrm{WH}, 29 ; P<.001)$ and urticarial/erythematous $(\mathrm{NWH}$, 3; $\mathrm{WH}, 13 ; P=.02)$ lesions. Follicular etiologies included acne $(\mathrm{NWH}, 1 ; \mathrm{WH}, 6 ; P=.12)$, folliculitis $(\mathrm{NWH}, 5$; $\mathrm{WH}, 2 ; P=.45)$, hidradenitis suppurativa $(\mathrm{NWH}, 2$; WH, 11; $P=.02)$, and pilonidal and sebaceous cysts $(\mathrm{NWH}, 1 ; \mathrm{WH}, 10 ; P=.01)$. Allergic urticaria dominated the urticarial/erythematous category $(\mathrm{NWH}, 3 ; \mathrm{WH}, 11$; $P=.06$ ), though there were $2 \mathrm{WH}$ presentations of diffuse erythema and skin peeling.

Another substantial proportion of cutaneous etiologies were due to trauma or chronic wounds. Significantly more traumatic injuries presented in NWH patients vs WH patients (36 vs 31; $P=.04$ ). Trauma included human or dog bites (NWH, 5; WH, 4), sunburns (NWH, 3; $\mathrm{WH}, 0)$, other burns (NWH, 11; WH, 13), abrasions and lacerations $(\mathrm{NWH}, 16 ; \mathrm{WH}, 3 ; P=.004)$, and foreign bodies (NWH, 1; WH, 1). Wounds consisted of chronic wounds such as those due to diabetes mellitus (foot ulcers) or immobility (sacral ulcers); numbers were similar between groups.

Looking at location, NWH patients had more pathology on the feet $(\mathrm{NWH}, 62 ; \mathrm{WH}, 39 ; P=.02)$, whereas $\mathrm{WH}$ patients had more disseminated multiregional 
TABLE 2. Morphology and Location of Cutaneous Diagnoses ${ }^{a, b}(\mathrm{~N}=842)$

\begin{tabular}{|c|c|c|c|}
\hline Cutaneous diagnosis & NWH & WH & $P$ value \\
\hline \multicolumn{4}{|l|}{ Morphology, $n$} \\
\hline Infectious & 212 & 150 & $<.001$ \\
\hline Bacterial & 127 & 100 & .04 \\
\hline Viral & 15 & 14 & .85 \\
\hline Fungal & 37 & 28 & .25 \\
\hline Infestation & 33 & 8 & $<.001$ \\
\hline Noninfectious inflammatory & 48 & 85 & $<.001$ \\
\hline Neutrophilic & 1 & 5 & .22 \\
\hline Follicular & 9 & 29 & 2 \\
\hline Urticaria/erythema & 3 & 13 & \\
\hline Panniculitis & 0 & 2 & \\
\hline Papulosquamous & 33 & 31 & 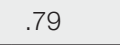 \\
\hline Autoimmune/connective tissue & 2 & 5 & .45 \\
\hline Neoplasm & 6 & 16 & .03 \\
\hline Benign & 4 & 9 & .26 \\
\hline Malignant & 2 & 7 & .18 \\
\hline Trauma and wounds & 62 & 65 & .49 \\
\hline Trauma & 36 & & .04 \\
\hline Wounds & 26 & & .28 \\
\hline Drug-related eruptions & 5 & 27 & $<.001$ \\
\hline Vascular & 24 & 21 & .65 \\
\hline Inflammatory & 10 & 8 & .63 \\
\hline Noninflammatory & 14 & 13 & .84 \\
\hline Pruritic & & 13 & .52 \\
\hline Pigmented & & 3 & .65 \\
\hline Bullous & & 12 & .67 \\
\hline Autoimmune & & 2 & 1.00 \\
\hline Nonautoimmune & 9 & 10 & 1.00 \\
\hline Neuropsychiatric & 12 & 5 & .09 \\
\hline Other & 30 & 34 & .60 \\
\hline Unknown, unspec & 22 & 19 & .63 \\
\hline Miscellaneous $^{\mathrm{C}}$ & 8 & 15 & .14 \\
\hline \multicolumn{4}{|l|}{ Location, $\mathrm{n}$} \\
\hline Disseminated, multiregional & 55 & 75 & .05 \\
\hline Head and neck & 42 & 51 & .23 \\
\hline Leg to ankle & 87 & 78 & .43 \\
\hline Shoulder to wrist & 64 & 48 & .10 \\
\hline Hand & 29 & 22 & .31 \\
\hline Foot & 62 & 39 & .02 \\
\hline Back, trunk, and abdomen & 28 & 40 & .13 \\
\hline Buttock, genitals & 54 & 68 & .17 \\
\hline
\end{tabular}

Abbreviations: $\mathrm{NWH}$, without housing; $\mathrm{WH}$, with housing.

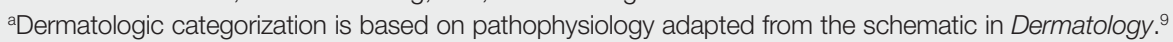

bIncludes chief concerns and secondary diagnoses.

cMiscellaneous included callus ( $\mathrm{NWH}, 4 ; \mathrm{WH}, 7)$, xerosis $(\mathrm{NWH}, 2 ; \mathrm{WH}, 5)$, polymorphous eruption of pregnancy (NWH, 1), cutaneous amyloidosis $(\mathrm{NWH}, 1)$, foreign-body granulomatous dermatitis $(\mathrm{WH}, 1)$, keloid $(\mathrm{WH}, 1)$, and acquired perforating dermatitis $(\mathrm{WH}, 1)$. 
concerns (NWH, 55; WH, 75; $P=.05$ ). No one body location was notably more likely to warrant a chief concern.

For clinical outcomes, more $\mathrm{WH}$ patients received a consultation of any kind (NWH, 171; WH, 217; $P<.001)$, consultation to dermatology (NWH, 49; WH, 87; $P<.001)$, and consultation to surgery (NWH, 64; WH, 110; $P<.001$ ) (Table 3 and Figure). More outpatient referrals to dermatology were made for $\mathrm{WH}$ patients $(\mathrm{NWH}, 61 ; \mathrm{WH}$, $82 ; P=.05)$. Notably, NWH patients presented for $80 \%$ fewer hospital follow-up appointments (NWH, 11; $\mathrm{WH}, 55 ; \mathrm{P}<.001)$. It is essential to note that these findings were not affected by self-reported race or ethnicity. Results remained significant when broken into cohorts consisting of patients with and without skin of color.

\section{Comment}

Cutaneous Concerns in NWH Patients-Although cutaneous disease has been reported to disproportionately affect NWH patients, ${ }^{10}$ in our cohort, NWH patients had fewer cutaneous chief concerns than WH patients. However, without comparing with all patients entering the ED at $\mathrm{UMC}$, we cannot make a statement on this claim. We do present a few reasons why NWH patients do not have more cutaneous concerns. First, they may wait to present with cutaneous disease until it becomes more severe (eg, until chronic wounds have progressed to infections). Second, as discussed in depth by Hollestein and Nijsten, ${ }^{3}$ dermatologic disease may be a major contributor to the overall count of disability-adjusted life years but mayplay a minor role in individual disability. Therefore, skin disease often is considered less important on an individual basis, despite substantial psychosocial burden, leading to further stigmatization of this vulnerable population and discouraged care-seeking behavior, particularly for

\section{TABLE 3. Clinical Workup ( $\mathrm{N}=842)$}

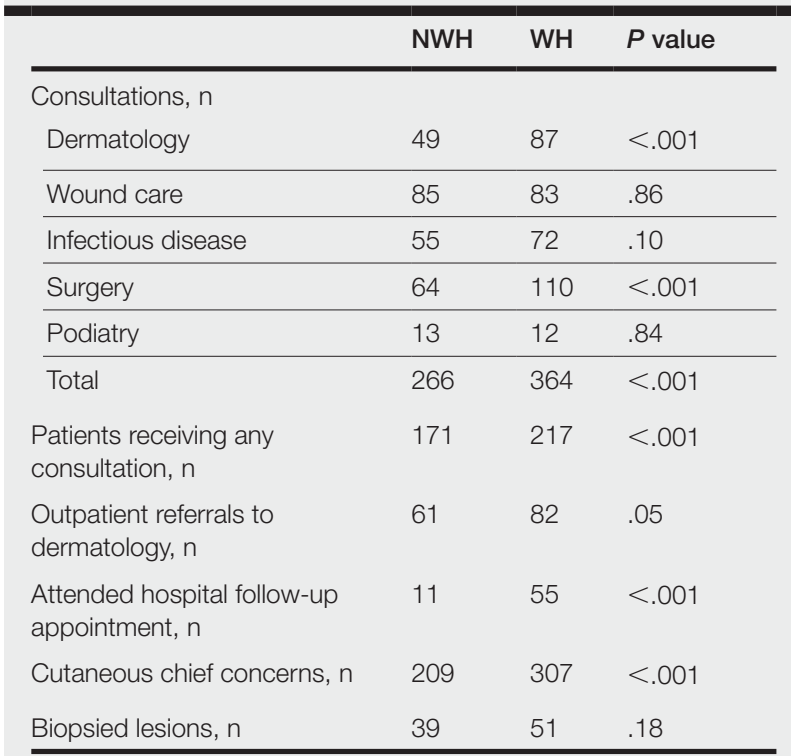

Abbreviations: $\mathrm{NWH}$, without housing; $\mathrm{WH}$, with housing.

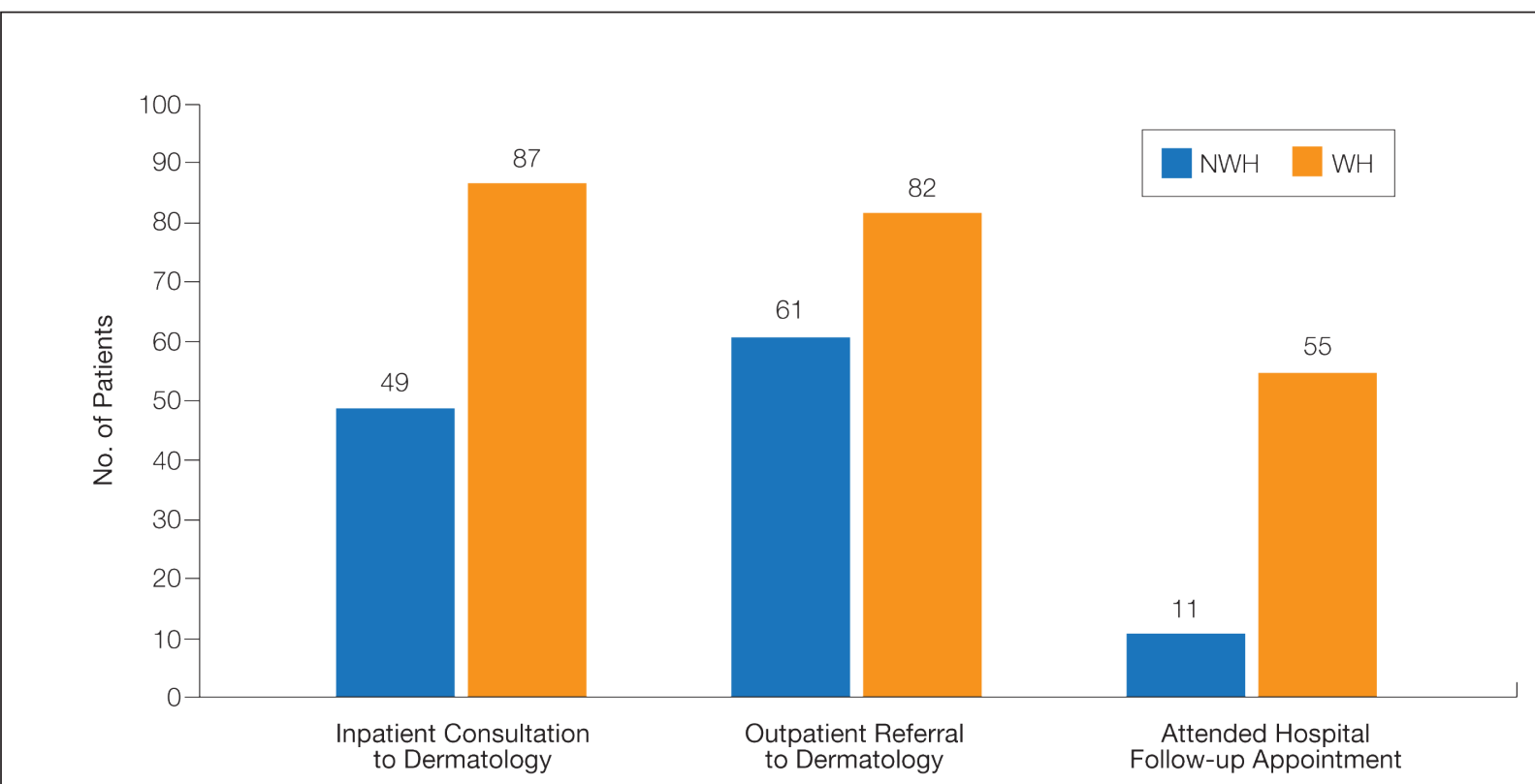

Differences in dermatologic care between those with housing $(\mathrm{WH})$ and without housing $(\mathrm{NWH})$. The number of patients receiving consultation, referral, or hospital follow-up after presenting to the emergency department and receiving a cutaneous or dermatologic diagnosis are presented. All results are statistically significant $(P<.001)$. 
noninfectious inflammatory eruptions, which were notably more present in WH individuals. Third, fewer dermatologic lesions were reported on NWH patients, which may explain why all $3 \mathrm{WH}$ pigmented lesions were diagnosed after presentation with a noncutaneous concern (eg, headache, anemia, nausea).

Infectious Cutaneous Diagnoses-The increased presentation of infectious etiologies, especially bacterial, is linked to the increased numbers of IVDUs reported in $\mathrm{NWH}$ individuals as well as increased exposure and decreased access to basic hygienic supplies. Intravenous drug use acted as an effect modifier of infectious etiology diagnoses, playing a major role in both $\mathrm{NWH}$ and WH cohorts. Although Black and Hispanic individuals as well as individuals with low socioeconomic status have increased proportions of skin cancer, there are inadequate data on the prevalence in $\mathrm{NWH}$ individuals. ${ }^{4} \mathrm{We}$ found no increase in malignant dermatologic processes in $\mathrm{NWH}$ individuals; however, this may be secondary to inadequate screening with a total body skin examination.

Clinical Workup of NWH Patients-Because most $\mathrm{NWH}$ individuals present to the ED to receive care, their care compared with WH patients should be considered. In this cohort, WH patients received a less extensive clinical workup. They received almost half as many dermatologic consultations and fewer outpatient referrals to dermatology. Major communication barriers may affect NWH presentation to follow-up, which was drastically lower than WH individuals, as scheduling typically occurs well after discharge from the ED or inpatient unit. We suggest a few alterations to improve dermatologic care for NWH individuals:

- Consider inpatient consultation for serious dermatologic conditions-even if chronic-to improve disease control, considering that many barriers inhibit follow-up in clinic.

- Involve outreach teams, such as the Assertive Community Treatment teams, that assist individuals by delivering medicine for psychiatric disorders, conducting total-body skin examinations, assisting with wound care, providing basic skin barrier creams or medicaments, and carrying information regarding outpatient follow-up.

- Educate ED providers on the most common skin concerns, especially those that fall within the noninfectious inflammatory category, such as hidradenitis suppurativa, which could easily be misdiagnosed as an abscess.

Future Directions-Owing to limitations of a retrospective cohort study, we present several opportunities for further research on this vulnerable population. The severity of disease, especially infectious etiologies, should be graded to determine if $\mathrm{NWH}$ patients truly present later in the disease course. The duration and quality of housing for NWH patients could be categorized based on living conditions (eg, on the street vs in a shelter). Although the findings of our NWH cohort presenting to the ED at UMC provide helpful insight into dermatologic disease, these findings may be disparate from those conducted at other locations in the United States. University Medical Center provides care to mostly subsidized insurance plans in a racially diverse community. Improved outcomes for the NWH individuals living in New Orleans start with obtaining a greater understanding of their diseases and where disparities exist that can be bridged with better care.

Acknowledgment-The dataset generated during this study and used for analysis is not publicly available to protect public health information but is available from the corresponding author on reasonable request.

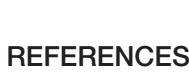

1. Fazel S, Geddes JR, Kushel M. The health of homeless people in highincome countries: descriptive epidemiology, health consequences, dol:10.1016/S0140-6736(14)61132-6

2. Contag C, Lowenstein SE, Jain S, et al. Survey of symptomatic dermatologic disease in homeless patients at a shelterbased clinic. Our Dermatol Online. 2017;8:133-137. doi:10.7241 lourd.20172.37

3. Hollestein LM, Nijsten T. An insight into the global burden of skin diseases. J Invest Dermatol. 2014;134:1499-1501. doi:10.1038 /jid.2013.513

4. Buster KJ, Stevens EI, Elmets CA. Dermatologic health disparities. Dermatol Clin. 2012;30:53-59. doi:10.1016/j.det.2011.08.002

5. Grossberg AL, Carranza D, Lamp K, et al. Dermatologic care in the homeless and underserved populations: observations from the Venice Family Clinic. Cutis. 2012;89:25-32.

6. Mackelprang JL, Graves JM, Rivara FP. Homeless in America: injuries treated in US emergency departments, 2007-2011. Int J Inj Contr Saf Promot. 2014;21:289-297. doi:10.1038/jid.2014.371

7. Chen CL, Fitzpatrick L, Kamel H. Who uses the emergency department for dermatologic care? a statewide analysis. J Am Acad Dermatol. 2014;71:308-313. doi:10.1016/j.jaad.2014.03.013

8. Stratigos AJ, Stern R, Gonzalez E, et al. Prevalence of skin disease in a cohort of shelter-based homeless men. J Am Acad Dermatol. 1999;41:197-202. doi:10.1016/S0190-9622(99)70048-4

9. Bolognia JL, Jorizzo JL, Schaffer JV, eds. Dermatology. 3rd ed. Elsevier; 2012.

10. Badiaga S, Menard A, Tissot Dupont H, et al. Prevalence of skin infections in sheltered homeless. Eur J Dermatol. 2005; 15:382-386. 